\title{
A study on holonic manufacturing systems and its application to real time scheduling problems
}

\author{
Nobuhiro Sugimura ${ }^{1}$, Toshimichi Moriwaki ${ }^{2}$, and \\ Keiichi Hozumi ${ }^{3}$ \\ ${ }^{1}$ College of Eng., Osaka Prefecture University, \\ Gakuencho, Sakai, Osaka 593, Japan, \\ Tel:+81-722-52-1161, Fax:+81-722-59-3340 \\ E-mail:sugimura@center.osakafu-u.ac.jp, \\ ${ }^{2}$ Faculty of Eng., Kobe University, \\ Rokkodai, Nada, Kobe 657, Japan, \\ ${ }^{3}$ ShiMaywa Industries Ltd., \\ 6-107, Tachikano, Takarazuka, 663, Japan.
}

\begin{abstract}
The objective of the present research is to develop an holonic architecture of manufacturing systems. A concept of the holonic structure of manufacturing systems are discussed, and an object oriented modeling method is proposed to represent the data and the activities of the holonic components of the manufacturing systems, such as workpieces, workstations, machine tools, AGVs and so on. Object oriented models are developed to represent simulation models of the holonic manufacturing system for the mechanical products and one for the composite parts of the aircrafts. Prototypes of the holonic real time scheduling systems are developed based on the simulation models, and case studies are carried out.
\end{abstract}

\section{Keywords}

Holonic manufacturing system, Real time scheduling, Object-oriented model, Simulation 


\section{INTRODUCTION}

With advances of manufacturing technologies in both software and hardware, automation of manufacturing systems in batch production has been much developed. The control structures of the manufacturing systems, such as FMSs (Flexible Manufacturing Systems) and FMCs (Flexible Manufacturing Cells), are generally hierarchical. The hierarchical control structure is suitable for economical and efficient batch productions in steady state, but not necessarily so for very small batch productions with dynamic changes in the volumes and varieties of the products.

Functions and capabilities of FA (Factory Automation) controllers have made much progress in the last decade, and individual controllers are now able to share information processing and decision making capabilities in the manufacturing systems. New architectures of the manufacturing systems are therefore proposed aiming at realizing more flexible control structures of the manufacturing systems which can cope with the dynamic changes in the volume and the variety of the products and also the unforeseen disruptions such as breakdown of equipment and interruption by high priority jobs (Okino, 1992), (Ueda, 1992), (Moriwaki, 1992), (Warnecke, 1993), (Iwata, 1994), (Wiendahl, 1994), (Sugimura, 1995), (Wyns, 1996), (Gou, 1997), (Bongaerts, 1997), (Arai, 1997).

Some researches have also been carried out for establishing modeling techniques of the manufacturing systems (Patankarm, 1995), (ISO TC 184, 1989), (AMICE, 1992), (Petrie, 1992). The models proposed provide basic framework for the modeling of the manufacturing systems including the holonic manufacturing systems.

The objective of the present research is to develop an holonic architecture of the manufacturing systems. A concept of the holonic structure of the manufacturing systems is discussed, and a holonic real time scheduling method is proposed in the paper. The main topics discussed in the paper are as follows;

(1) basic architecture of the holonic manufacturing system (HMS),

(2) object oriented modeling of HMS, and

(3) holonic real time production scheduling of the manufacturing systems for mechanical assemblies and composite parts.

\section{BASIC ARCHITECTURE OF HOLONIC MANUFACTURING SYSTEM}

The holonic manufacturing systems are composed of sets of autonomous and cooperative components called holonic components, which include such components as workstations, AGVs, storages and workpieces. The individual holonic components 
have functions of information processing, decision making and communication, and also material processing for the manufacturing.

Figure 1 shows a basic concept of the holonic manufacturing systems. The physical model describes the holonic components of the manufacturing systems and the flows of the materials in the systems. The logical model gives the holonic information processing and the decision making structures in the systems. As shown in the figure, the production control of the system is carried out through the decision making of the individual holonic components and their cooperations.

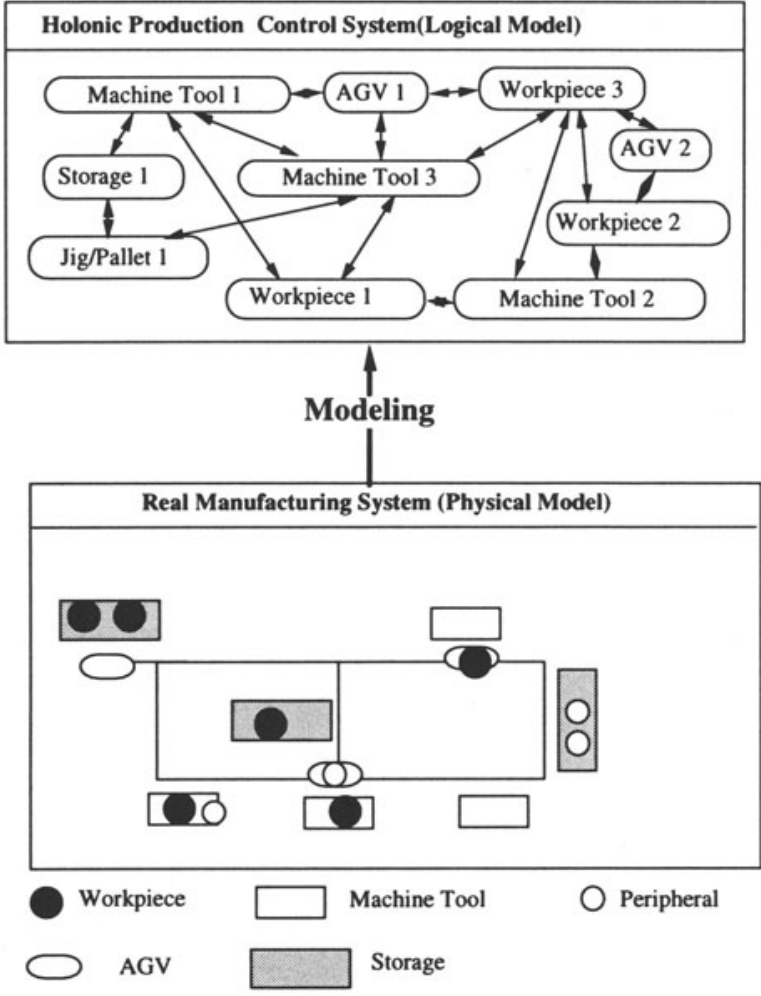

Figure 1 Holonic manufacturing systems

The holonic components of the manufacturing systems are basically divided into two classes based on their roles in the manufacturing processes; they are,

(a) Manufacturing equipment which transforms the blank materials to the finished products, and

(b) Workpieces which are transformed by the manufacturing equipment.

The manufacturing equipment includes all the equipment, such as machine tools, assembly stations, tools, jigs, AGVs, and storages. Blank materials, parts, subassemblies and assemblies are typical examples of the workpieces.

The manufacturing equipment and the workpieces are required to have holonic decision making functions to determine their own suitable manufacturing processes in the manufacturing systems on the basis of their status. The followings summarizes the important decision making functions of the manufacturing equipment and the workpieces; 
(a) Manufacturing equipment

1) Design and modification of manufacturing functions.

2) Operation planning and task planning.

3) Production scheduling.

4) Production planning and MRP (Material Requirement Program).

(b) Workpieces

1) Modification of their shapes, dimensions and tolerances.

2) Process planning.

3) Production scheduling.

4) Production planning and MRP.

\section{MODELING OF HOLONIC MANUFACTURING SYSTEMS}

An object oriented modeling technique is adopted to represent the holonic components which carry out the decision making functions for the production control of the manufacturing systems. The individual objects have both the data representing the status and the methods describing the decision making procedures.

Object oriented models are proposed to describe and to simulate the decision making activities and the communication activities of the holonic components of the manufacturing systems, based on the investigation of the manufacturing processes of the products. Two types of manufacturing systems are considered here; they are, a manufacturing system of mechanical assemblies, and a manufacturing system of composite parts of civil aircrafts. Schematic illustrations of these two types of manufacturing systems are shown in Figs. 2 and 3, respectively.

The manufacturing system for the mechanical assemblies consists of machining shops, assembly stations, storages and AGVs, and the manufacturing processes are carried out by the batch processing. The objects shown in Fig. 4 are needed to carry out the production control of the manufacturing systems. In the figure, the nodes show the objects, and the arcs give the is_a relations and the part_of relations among the objects. The objects are basically classified into four; they are, the workpieces, the manufacturing equipment, the manufacturing processes of the workpieces, and the operations.

The Manufacturing system for the composite parts is basically a line type system, however, parallel line processing and batch processing are included as shown in Fig. 3. The objects needed to represent the model of the manufacturing system are slightly different from the ones for the manufacturing system for the mechanical assemblies, however, the basic components and their relationships are same as ones in Fig. 4. 


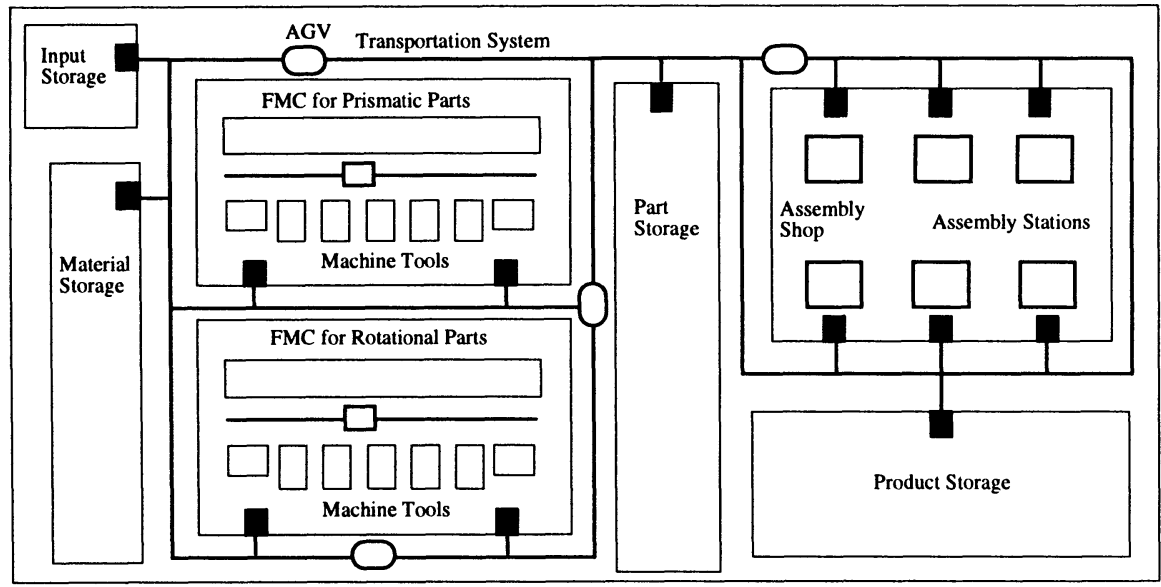

Figure 2 Manufacturing system for mechanical assemblies

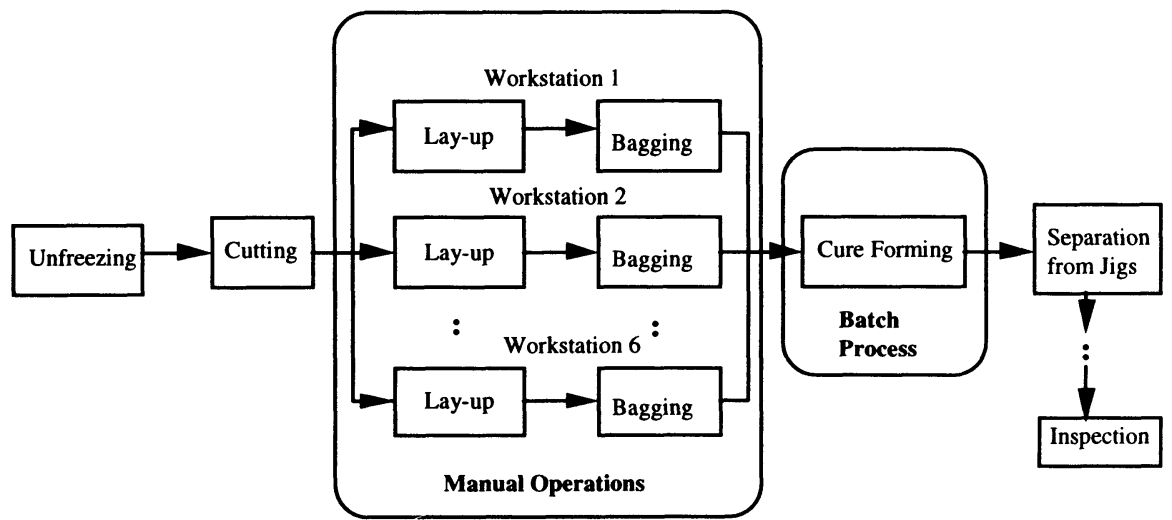

Figure 3 Manufacturing system for composite parts

\section{HOLONIC SCHEDULING SYSTEM}

\subsection{Decision Making Structure}

The concept of the holonic manufacturing systems is applied to a real time production scheduling systems which is an important problems in the production control. The real time scheduling means that the schedule is determined dynamically only when the status of the manufacturing systems is changed due to the progress of the manufacturing processes and the unforeseen events. Therefore, the scheduling systems only determine the schedules of the manufacturing equipment and the workpieces for very short time period. 


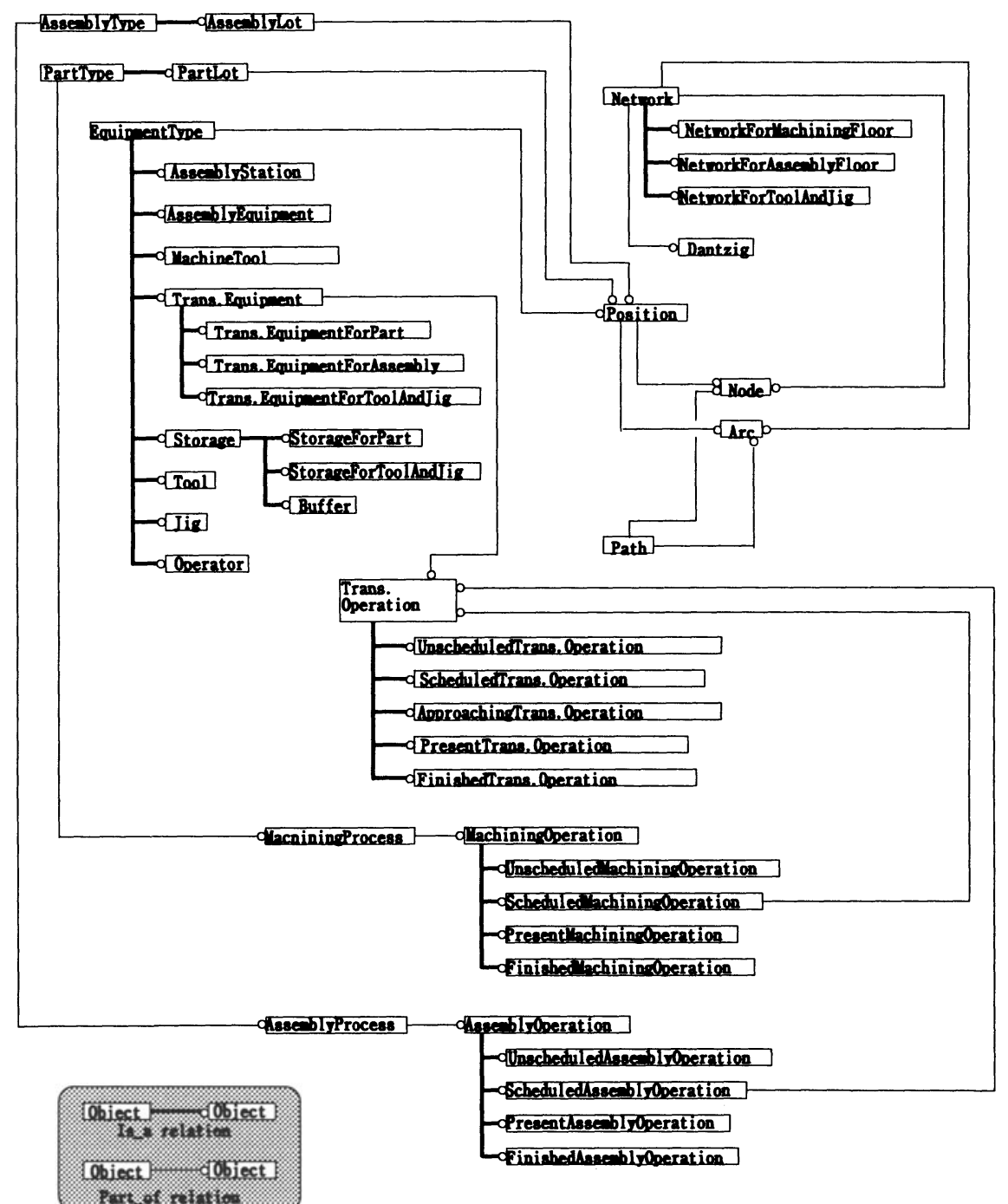

Figure 4 Objects and their relationships needed for production control

The scheduling systems consist of a set of holonic objects representing the workpieces and the manufacturing equipment, such as the machine tools, the workstations, and the jigs. The production schedule is determined based on the decision makings of the individual holonic objects and their communications. The decisions of the individual objects are made by applying the heuristic rules.

The procedure to determine the schedule is summarized in Fig. 5. The individual objects modify their status, in the first step, if one of the following events occurs; 


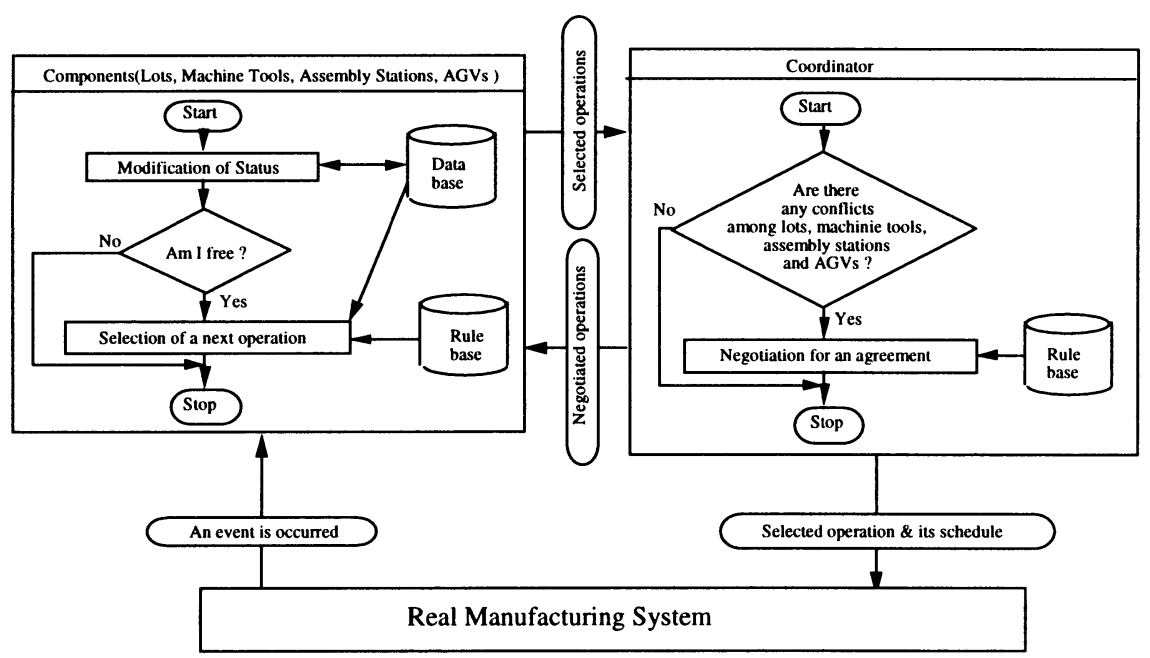

Figure 5 Scheduling processes

(1) an operation of a workpiece/lot is finished,

(2) a workpiece/lot is inputted to the manufacturing system,

(3) a equipment is broken down, or is recovered, and

(4) a status of a workpiece/lot is changed from the normal one to the high priority one.

In the second step, all the objects which are free at that time select suitable operations to be carried out in the next time period of the manufacturing systems based on their own criteria. The time period means the period between the time when one event occurs and one when another event occurs. The information about the selected operations are transmitted to the coordinator, and the conflicts among them are examined. If the conflicts are detected, the decisions of some objects are rejected and changed by the coordinator. After the coordination, the operations to be carried out in the next period of the manufacturing systems are scheduled. The procedures above mentioned are repeated, when the events occur.

\subsection{Scheduling Criteria of Components}

A rule based approach is applied in the research to describe the decision making criteria to select the operations to be carried out and to coordinate the conflicts. The rules of individual objects are described as the methods in the objects. The contents of the decision criteria of the objects are summarized in the followings.

(1) Lot object

Each lot object has the criteria to select a suitable manufacturing equipment which carries out the operation of the lot in the next time period of the manufacturing system. The basic criterion is as follows; 
- Select a manufacturing equipment which can finish the next operation of the lot at the earliest time. Where, the finishing time of the next operation is calculated from both the manufacturing time given in the manufacturing operation objects and the estimated transportation time by the AGVs.

The high priority lots must be finished their operations as soon as possible, therefore, the objects of the high priority lots have also the criteria to reserve the manufacturing equipment and the AGVs needed for the future operations of the lots. The criteria to reserve the manufacturing equipment and the AGVs are as follows;

- Select a manufacturing equipment which can finish the operation of the lot in the shortest time, and

- Select an AGV which can transport the lot in the shortest time.

(2) Manufacturing equipment objects

The manufacturing equipment objects have the criteria to select suitable operations to be carried out in the next time period, when they are free at that time. The criteria are summarized as follows;

- Select a operation of the high priority lots, if the high priority lot requires the operations which can be carried out by the manufacturing equipment, and

- Select a operation of the normal lots which can be finished in the shortest time.

(3) AGV objects

The decision criteria of the AGV objects are the rules to select suitable lots which the AGVs transport. The criteria are given as follows;

- Select a high priority lot, if the high priority lot requires the transportation operation, and

- Select a normal lot which requires the transportation operation at the earliest time.

(4) Coordinator object

The coordinator object coordinates the conflicts among the decisions of the lots, the manufacturing equipment and the AGVs. The basic rules of the coordinator object are given in the following;

- High priority is given to the operations of the high priority lots, and

- Select operations which are selected by both the lots and the manufacturing equipment.

\subsection{CASE STUDIES}

A prototype of holonic real time production scheduling system has been implemented in Smalltalk/Visual Works. Some case studies have been carried out to verify the feasibility of the holonic real time production scheduling method proposed here. In particular, emphasis has been given to clarifying the adaptability of 
the holonic system to the scheduling problems of the manufacturing system for the mechanical assemblies and one for the composite parts.

The system for the mechanical assemblies shown in Fig. 2 consists of an FMC composed of four vertical type machining centers and six turning centers, an assembly shop composed of two assembly stations, and a transportation \& storage system composed of two AGVs and four storages. One lot of an assembly and the part lots related to the assembly lot are inputted to the manufacturing systems for the case study. Figure 6 shows an example of schedule determined by the prototype system. In the figure, the vertical axis indicate the manufacturing equipment and the lots to be manufactured, and the horizontal axis shows the time. The individual line segments indicates the operations of the individual lots and their schedules. It can be found that the system can generate feasible schedules for the FMC, the assembly shop, and the transportation and storage system.

The other case studies have been carried out for the scheduling of the line production for the composite parts shown in Fig. 3. The manufacturing processes scheduled by the real time scheduling method are shown in Fig. 7. In the figure, the vertical axis indicate the lots to be manufactured, and the horizontal axis shows the time. The individual line segments indicates the operations of the individual lots and their schedules.

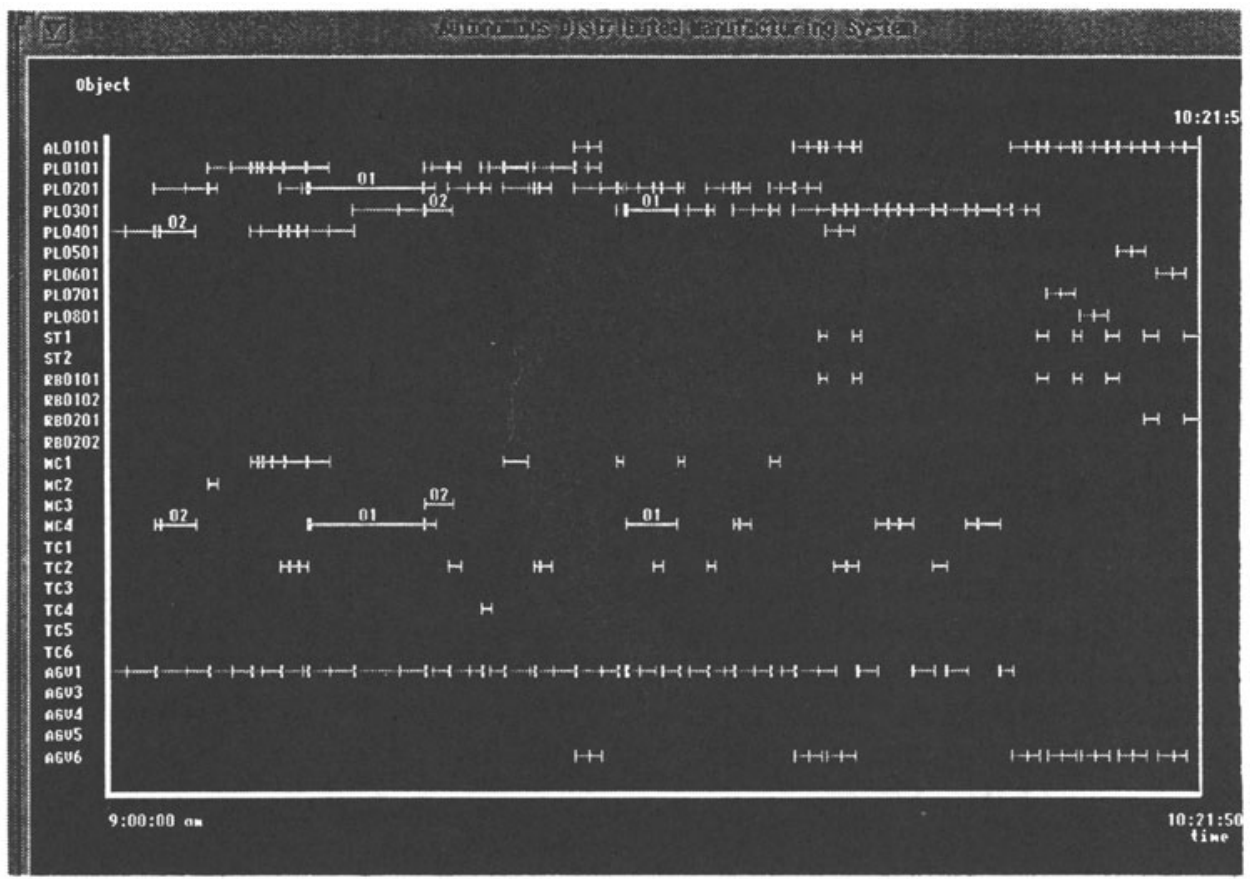

Figure 6 Example of schedule of manufacturing system for mechanical assemblies 


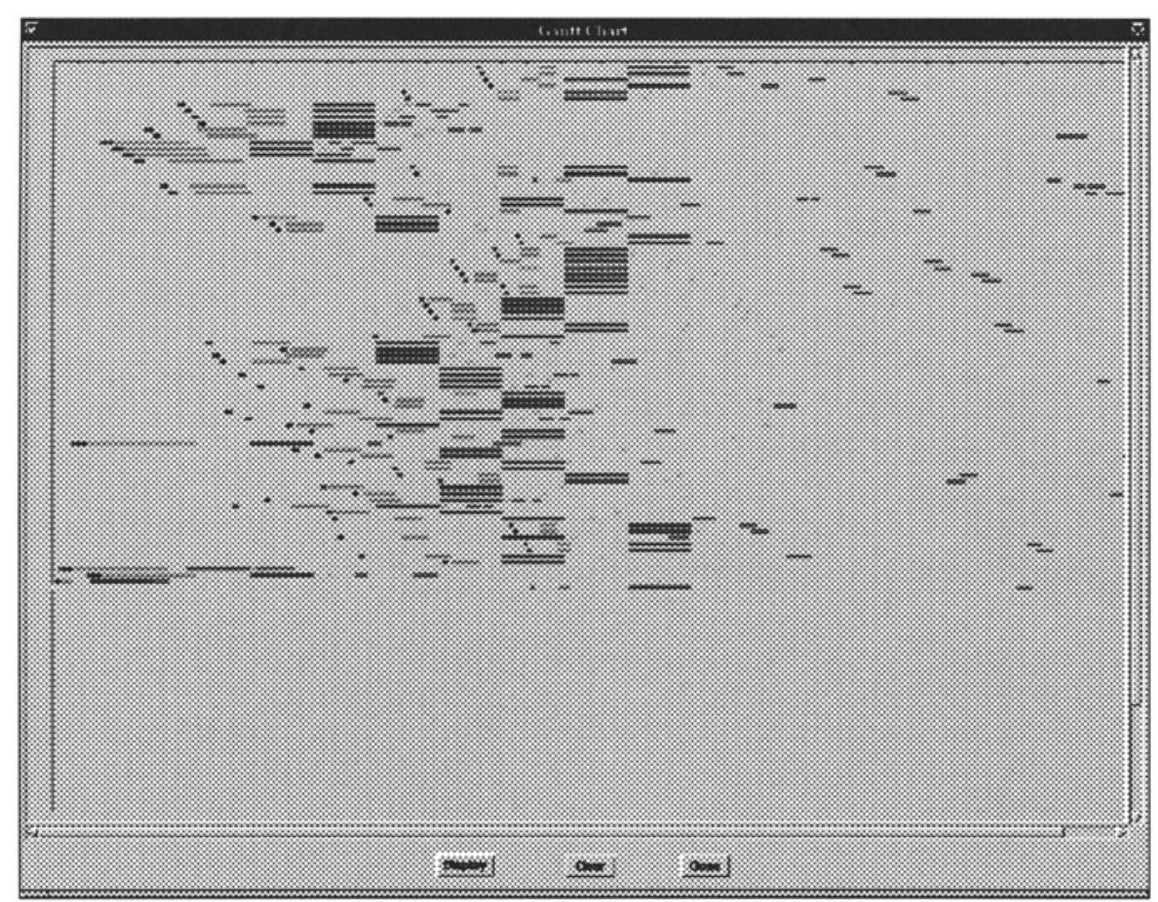

Figure 7 Example of schedule of manufacturing system for composite parts

It is shown, through the case studies, that the holonic real time scheduling methods proposed here can generate suitable manufacturing schedules for the manufacturing systems considered here.

\section{CONCLUSIONS}

A basic architecture of the holonic manufacturing systems was discussed, and an object oriented modeling technique was proposed aiming at describing the status and the activities of the holonic manufacturing systems. Prototypes of real time production scheduling system were implemented based on the holonic architecture. The following remarks are concluded.

(1) An object oriented modeling method is proposed to describe the information processing and decision making functions of the holonic components.

(2) Objects needed for the holonic real time production scheduling system are established, which represent the components such as the manufacturing equipment, the workpieces/lots, the processes and the operations.

(3) Prototype production scheduling systems have been implemented in Smalltalk/Visual Works and applied to some scheduling problems of the manufacturing system for the mechanical assemblies and one for the composite parts. 


\section{REFERENCES}

Okino, N. (1992). A Prototyping of Bionic Manufacturing System, Proc. of the ICOOMS '92, pp.297-302.

Ueda, K. (1992). An Approach to Bionic Manufacturing Systems Based on DNAType Information, Proc. of the ICOOMS '92, pp.303-308.

Moriwaki, T. and Sugimura, N. (1992).Object Oriented Modeling of Autonomous Distributed Manufacturing System and its Application to Real Time Scheduling, Proc. of the ICOOMS '92, pp.207-212.

Warnecke, H.J. (1993). The Fractal Enterprise, Springer-Verlag, New York. Iwata, K., et al. (1994). Random Manufacturing System: A New Concept of Manufacturing Systems for Production to Order, Annals of the CIRP, Vol. 43, No.1, pp.379-384.

Wiendahl, H.P. and Garlichs, R. (1994) Decentral Production Scheduling of Assembly Systems with Genetic Algorithm, Annals of the CIRP, Vol.43, No.1, pp.389-396.

Sugimura, N., et. al. (1995). Modeling of holonic manufacturing system and its application to real time scheduling, Proc. of the 27th Int. Seminar on Manufacturing Systems, pp.401-410.

Wyns, J., et. al. (1996). Workstation Architecture in Holonic Manufacturing Systems, Proc. of the 28th Int. Seminar on Manufacturing Systems, pp.220-231.

Gou, L., et. al. (1997).Holonic Manufacturing Scheduling; Architecture, Cooperation Mechanism, and Implementation, Proc. of IEEE/ASME Int. Conf. on Advanced Intelligent Mechatronics, pp.1-12.

Bongaerts, L. et. al. (1997), Schedule Execution in Holonic Manufacturing Systems, Proc. of the 29th CIRP Int. Seminar on Manufacturing Systems, pp.209214.

Arai, T., et. al. (1997). Holonic Storage: An Assembly and Storage Cell by Manipulation Using Environment, Proc. of the 29th CIRP Int. Seminar on Manufacturing Systems, pp.221-226.

Patankarm A. K. (1995). Enterprise Integration Modeling: A Review of Theory and Practice, Computer Integrated Manufacturing Systems, ButterworthHeinemann, pp.21-34.

ISO TC 184 (1989). Reference Model for Shop Floor Standards, ISO Technical Report 10314.

AMICE (1992). CIMOSA Project Deliverables - Mile-stone M-2 Part 1, ESPRIT Consortium AMICE, pp.9-10.

Petrie, C. J. (1992). Modeling Methodology: In Enterprise Integration Modeling, Proc. of the 1st Int. Conference, MIT Press.

\section{BIOGRAPHY}

Nobuhiro Sugimura received B.S. and M.S. degrees in engineering from Kobe University, Japan, in 1976 and 1978, respectively. He was a Research Associate 
from 1980 to 1987, and a Associate Professor from 1987 to 1994 at Faculty of Engineering, Kobe University. He received a ph. D degree in engineering from Osaka University, in 1985. Since 1994, he is currently a Full Professor at College of Engineering, Osaka Prefecture University. His research interests include, modeling and simulation of holonic manufacturing systems, CAD/CAM and product modeling for mechanical products, standardization of product model STEP, and modeling and design of machine tools.

Toshimichi Moriwaki received B.S. , M.S. and ph. D degrees in engineering from Kyoto University , Japan in 1966, 1968 and 1974 respectively. He was employed as a research associate at Mechanical Engineering Department, Kobe University in 1968, and promoted to Associate Professor and Full Professor in 1974 and 1985 respectively. He was a research engineer/Assistant Professor at McMaster University, Canada in 1975 and 1976. His research interests include intelligent manufacturing systems, intelligent machine tools, sensing technology in manufacturing, ultraprecision machine tools and machining process and human factors in manufacturing.

Keiichi Hozumi received B.S. in engineering from Kobe University, Japan, in 1970. $\mathrm{He}$ is working in Research \& Development Division of ShinMaywa Industries, Ltd. from 1970, which company products Aircraft, Factory Automation Related Products, Truck Bodies \& Equipment etc. in Japan. He had researched and developed control systems, sensors for Welding-robots and AC motors until 1985. Since 1986, his research interests are simulation and scheduling for manufacturing systems. Since 1997, he is a manager of Production engineering center in his company. He received a ph. D degree in engineering from Kobe University in 1997. 